\title{
Versatile Nature of Oxygen Vacancies in Bismuth Vanadate Bulk and (001) Surface
}

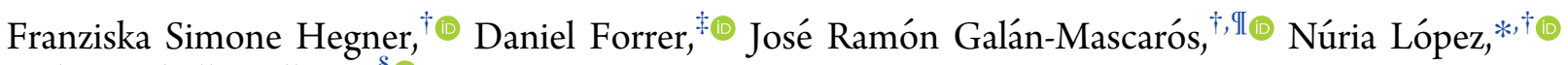 \\ and Annabella Selloni* ${ }^{*} \odot$
${ }^{\dagger}$ Institute of Chemical Research of Catalonia (ICIQ), The Barcelona Institute of Science and Technology (BIST), Avinguda Paisos Catalans 16, 43007 Tarragona, Spain
${ }^{\ddagger}$ ICMATE-CNR and INSTM, Via F. Marzolo 1, 35131 Padua, Italy
IICREA, Passeig Lluís Companys 23, 08010 Barcelona, Spain
${ }^{\S}$ Princeton University, Department of Chemistry, New Jersey 08544, United States

Supporting Information

\begin{abstract}
Bismuth vanadate $\left(\mathrm{BiVO}_{4}\right)$ has emerged as one of the most promising photoanode materials for solar fuel production. Oxygen vacancies play a pivotal role in the photoelectrochemical efficiency, yet their electronic nature and contribution to $n$-type conductivity are still under debate. Using first-principles calculations, we show that oxygen vacancies in $\mathrm{BiVO}_{4}$ have two distinguishable geometric configurations characterized by either undercoordinated, reduced $\mathrm{V}^{\mathrm{N}} \mathrm{O}_{3}$ and $\mathrm{Bi}^{\mathrm{II}} \mathrm{O}_{7}$ subunits or a $\mathrm{V}^{\mathrm{IV}}-\mathrm{O}-\mathrm{V}^{\mathrm{IV} / \mathrm{V}}$ bridge (split vacancy), quenching the oxygen vacancy site. While both configurations have similar energies in the bulk, the (001) subsurface acts like an energetic sink that stabilizes the split oxygen vacancy by $\sim 1 \mathrm{eV}$. The barrierless creation of a bridging $\mathrm{V}_{2} \mathrm{O}_{7}$ unit allows for partial electron delocalization throughout the near-surface region, consistent with recent experimental observations indicating that $\mathrm{BiVO}_{4}(001)$ is an electron-rich surface.
\end{abstract}

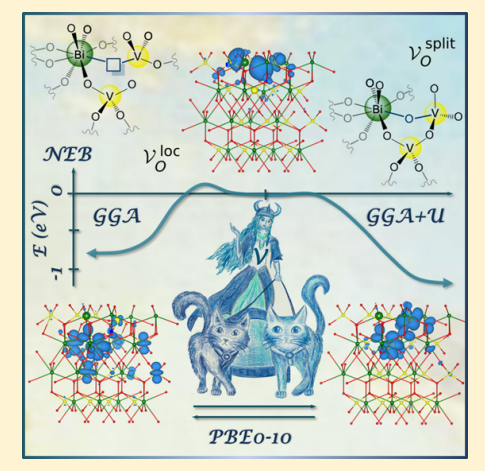

$\mathrm{O}$ ver the last two decades, bismuth vanadate, $\mathrm{BiVO}_{4}$, has become one of the most important photoanode materials for light-driven water splitting. ${ }^{1-4}$ Among the class of $n$-type metal oxide semiconductors, it has demonstrated the highest performance in photoelectrochemical (PEC) water oxidation to date. ${ }^{4,5}$ In addition to its suitable band gap (2.4$2.5 \mathrm{eV})$ and valence band edge position ( $2.8 \mathrm{~V}$ vs RHE), it also shows superior electron-hole separation and hole mobility. ${ }^{2,3}$ Notwithstanding, its efficiency is hindered by fast surface recombination ${ }^{6,7}$ and low electron mobility. 8,9 The latter is typically ascribed to local lattice distortions forming small polarons, which limit electronic conduction. ${ }^{9-15}$

Various experimental studies have reported that the PEC performance of $\mathrm{BiVO}_{4}$ is improved by the formation of oxygen vacancies $\left(\mathcal{V}_{\mathrm{O}}\right.$ 's $) .{ }^{16-18}$ The effect of $\mathcal{V}_{\mathrm{O}}$ 's is typically ascribed to enhancing $n$-type conductivity and therefore electronic transport, as in many metal oxide semiconductors. ${ }^{18-22}$ Removal of an oxygen atom leaves two excess electrons, which may be either mobile or (partially) bound to the vacancy. In the latter case, the metal atoms ( $\mathrm{V}$ and $\mathrm{Bi}$ ) surrounding the vacancy are expected to be reduced. For being effective $n$-type dopants, the electronic states created by $\mathcal{V}_{\mathrm{O}}$ 's have to lie close to the conduction band minimum (CBM), from where they can be thermally excited and act as shallow donors. Otherwise, if deep, localized intragap states are formed, electrons are trapped and hardly participate in electron conduction. ${ }^{23}$ Other possible roles of oxygen vacancies in
$\mathrm{BiVO}_{4}$ are related to increasing the number of accessible surface sites, thus favoring adsorption of oxidation reaction intermediates, decreasing the band gap, and enhancing interfacial charge transfer to the electrolyte. ${ }^{24,25}$ However, by forming electron-hole recombination centers and hindering charge-separation, $\mathcal{V}_{\mathrm{O}}$ 's can also have a detrimental effect on the PEC activity. ${ }^{23,25,26}$ Despite their important impact on the behavior of $\mathrm{BiVO}_{4}$, conclusive studies on oxygen vacancies are still limited and controversial. ${ }^{14,27}$ Although reduced V sites on the $\mathrm{BiVO}_{4}$ surface were directly observed and attributed to surface $\mathcal{V}_{\mathrm{O}}{ }^{\prime},{ }^{28}$ to our knowledge an unambiguous experimental determination of their nature and location with respect to the surface has not yet been reported.

While several theoretical studies of vacancy formation in the bulk suggest that $\mathcal{V}_{\mathrm{O}}$ 's generate shallow intragap states, ${ }^{21,22,29}$ more recent work indicates that the $\mathcal{V}_{\mathrm{O}}$-induced electronic states lie deep in the band gap, where they cannot contribute significantly to $n$-type conductivity ${ }^{27,30}$ but may act as polaronic carriers. ${ }^{14,15}$ Only few reports have considered $V_{\mathrm{O}}$ 's on the surface of $\mathrm{BiVO}_{4} \cdot{ }^{14,25,31}$ It was suggested that those $\mathcal{V}_{\mathrm{O}}$ 's have a beneficial influence on water oxidation on the (001) surface because of better adsorption of reaction 


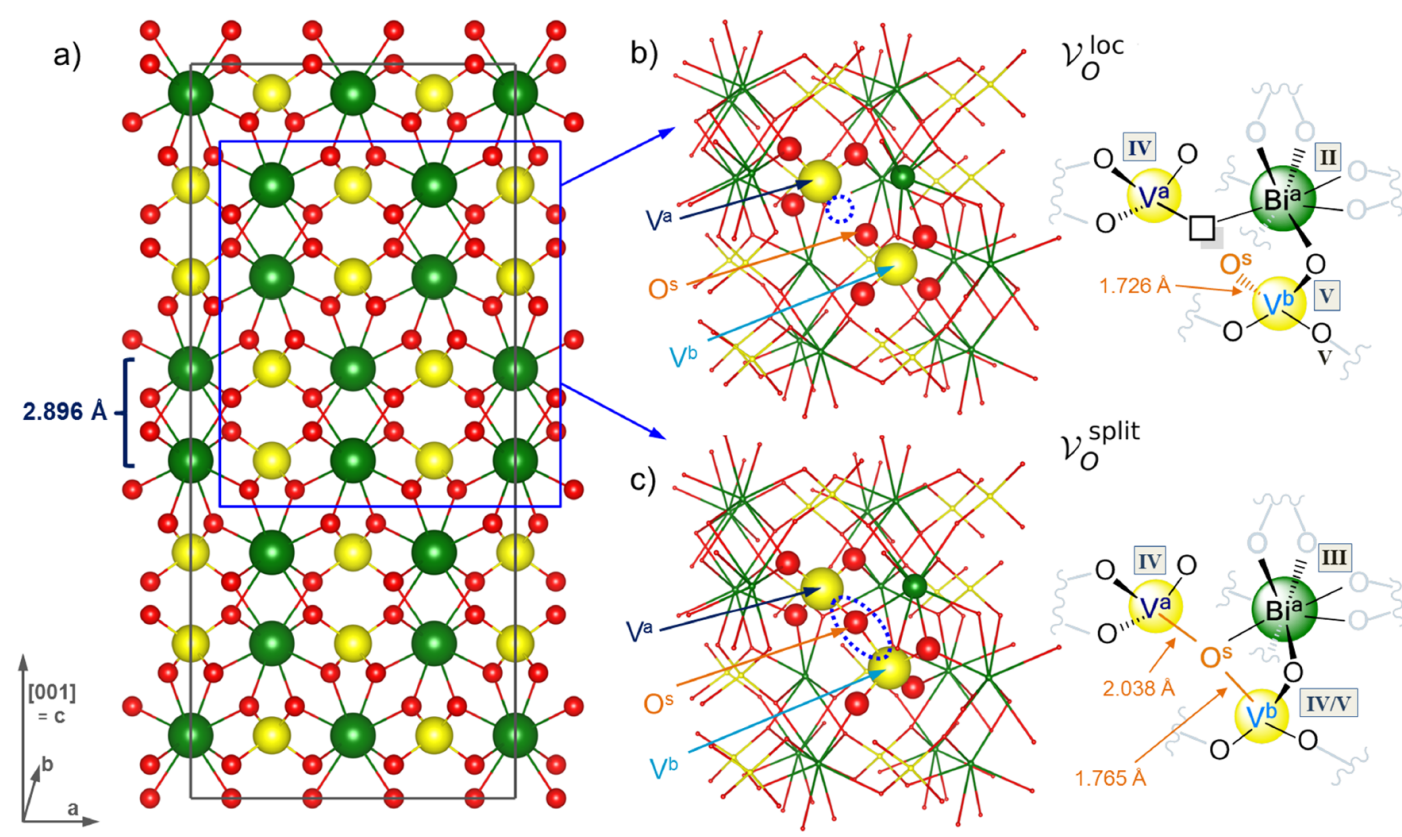

Figure 1. (a) Pristine bulk $2 \times 2 \times 2$ supercell of $\mathrm{BiVO}_{4}$ as used in the simulations (viewed from the [010] direction). (b) $\mathcal{V}_{\mathrm{O}}^{\text {loc }}:$ Localized oxygen vacancy forming undercoordinated $\mathrm{V}^{\mathrm{a}} \mathrm{IV}_{3}$ and $\mathrm{Bi}^{\mathrm{a}}{ }^{\mathrm{II}} \mathrm{O}_{7}$. (b) $\mathcal{V}_{\mathrm{O}}^{\text {split }}$ : Split vacancy between $\mathrm{V}^{\mathrm{a} \text { IV }}$ and $\mathrm{V}^{\mathrm{b}} \mathrm{IV} / \mathrm{V}$ that results in a $\mathrm{V}_{2} \mathrm{O}_{7}$ dimer with a shared (or split) oxygen, labeled $\mathrm{O}^{\mathrm{s}}$. Reported distances were computed with the PBEsol functional. Classical oxidation states of the relevant atoms are indicated. Color code: $\mathrm{Bi}, \mathrm{V}$, and $\mathrm{O}$ are green, yellow, and red, respectively (visualized with VESTA ${ }^{39}$ ).

intermediates and improved charge transfer on intrinsic ${ }^{24}$ and W-doped ${ }^{25} \mathrm{BiVO}_{4}$.

Herein, we used first-principles density functional theory (DFT) calculations to study the role of $\mathcal{V}_{\mathrm{O}}$ 's in the bulk and on the most stable (001) surface of $\mathrm{BiVO}_{4}$. Our results show that $\mathrm{O}$ removal leads to different possible geometric arrangements with distinct electron structures, as shown in Figure 1.

DFT calculations were performed with the Quantum Espresso (QE) software package, ${ }^{32,33}$ as described in the Supporting Information. The vacancy formation energies $E_{\mathrm{f}}^{\mathrm{O}}$ were referenced to the chemical potential of oxygen in the gas phase (Supporting Information, section S1.2). It was recently found that the used DFT functional has a large impact on the positions of $\mathcal{V}_{\mathrm{O}}$-induced energy levels and hence their contribution to conductivity. ${ }^{15,30}$ In order to address such dependency of the electronic structure, we used functionals of different levels of accuracy: GGA (PBEsol), GGA+U (PBEsol $+\mathrm{U}$ with $U=2.5 \mathrm{eV}^{25,34}$ (Supporting Information, section S1.3)), and hybrid functionals (PBE0-10*/PBE0-10**) with an optimized fraction (10\%) of exact exchange (Supporting Information, section S1.4). ${ }^{35}$ For the hybrids (PBE0-10*/ PBE0-10**), single-point calculations were carried out on structures, from prior optimization with PBEsol (PBE0-10*) or PBEsol+U (PBE0-10**). All structures have been uploaded to the ioChemBD ${ }^{36}$ database and can be accessed under the DOI 10.19061/iochem-bd-1-133. ${ }^{37}$

The photocatalytically active polymorph of bismuth vanadate exhibits a monoclinic scheelite phase, $m s-B \mathrm{BVO}_{4}$ ( $m s$ is henceforth omitted). ${ }^{1,3}$ It contains $\mathrm{BiO}_{8}$ units and $\mathrm{VO}_{4}$ tetrahedra, which are linked by bridging oxygens ( $\mathrm{Bi}-\mathrm{O}-$ $\mathrm{V})$ and stacked along the main [001] axis of the unit cell with interplanar distances of $2.896 \pm 0.002 \AA$, as shown in Figure 1a. ${ }^{38}$
The flexibility of the structure, as well as the versatile chemistry of the tetrahedral $\mathrm{VO}_{4}$ subunits, allows for the formation of two distinct types of oxygen vacancies: ${ }^{14,40,41}$ a lattice-centered or localized vacancy $\mathcal{V}_{\mathrm{O}}^{\text {loc }}$, leaving undercoordinated, reduced $\mathrm{V}^{\mathrm{a}}{ }^{\mathrm{IV}} \mathrm{O}_{3}$ and $\mathrm{Bi}^{\mathrm{a}}{ }^{\text {II }} \mathrm{O}_{7}$ (Figure $1 \mathrm{~b}$ ), and a bridged or split vacancy, $\mathcal{V}_{\mathrm{O}}^{\text {split }}$, where one $\mathrm{O}$ atom $\left(\mathrm{O}^{\mathrm{s}}\right)$ is at the bond-center of a $\mathrm{V}^{\mathrm{a}}-\mathrm{O}^{\mathrm{s}}-\mathrm{V}^{\mathrm{b}}$ bridge (Figure 1c). ${ }^{42,43}$ The latter is created when the neighboring $\mathrm{V}^{\mathrm{b}} \mathrm{O}_{4}$ orthovanadate from the adjacent layer rotates and moves toward the undercoordinated $\mathrm{V}^{\mathrm{a}} \mathrm{O}_{3}$, so that its oxygen can coordinate to the vacant site, forming $\mathrm{V}_{2}^{\mathrm{IV} / \mathrm{V}} \mathrm{O}_{7}$ pyrovanadate and reestablishing the 8-fold coordination sphere of $\mathrm{Bi}$ and its oxidation state of III. This quenches the vacancy and leads to a concomitant (partial) electron delocalization as discussed later in the text and in more detail in the Supporting Information (section S2.2). In such a distortion, the $\mathrm{V}^{\mathrm{a}}-\mathrm{O}^{\mathrm{s}}$ distance decreases from $2.732 \AA(2.882 \AA)$ to $2.038 \AA$ (2.152 $\AA$ ), while the $\mathrm{V}^{\mathrm{b}}-\mathrm{O}^{\mathrm{s}}$ distance increases by only about $0.039 \AA$ ( $0.031 \AA$ ) with PBEsol (PBEsol+U) (see Table $\mathrm{S} 2$ for the changes in bond length). Condensation of $\mathrm{VO}_{4}$ orthovanadates to produce polyoxovanadates is well-known in solution ${ }^{41}$ and has been identified also in solids between the crystalline layers at the $\mathrm{V}_{2} \mathrm{O}_{5}(001)$ surface, reducing the cost of $\mathcal{V}_{\mathrm{O}}$ formation. ${ }^{44}$ However, to the best of our knowledge, $\mathcal{V}_{\mathrm{O}}^{\text {split }}$ formation in $\mathrm{BiVO}_{4}$ and its impact on the electronic structure has not yet been investigated.

For bulk $\mathrm{BiVO}_{4}$ our calculations yield similar formation energies $E_{\mathrm{f}}^{\mathrm{O}}$ for both $\mathcal{V}_{\mathrm{O}}^{\text {split }}$ and $\mathcal{V}_{\mathrm{O}}^{\text {loc }}$ vacancies. The differences between them lie in a range of $0.2 \mathrm{eV}$ with a transition barrier $\lesssim 0.2 \mathrm{eV}$ depending on the employed DFT functional (see Figures $2 \mathrm{c}$ and $3 \mathrm{~b}$ and Table S3). We therefore expect both types of vacancies to be present in synthesized $\mathrm{BiVO}_{4}$, because during the calcination process at elevated temperatures 

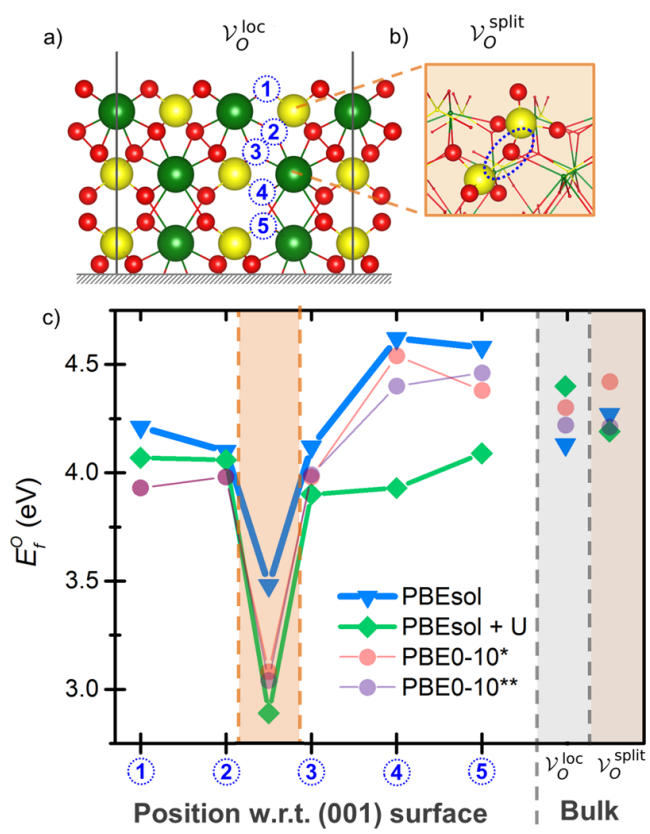

Figure 2. (a) Pristine $\mathrm{BiVO}_{4}(001)$ surface (viewed from [010] direction), showing the positions of the $\mathrm{O}$ atoms that are removed to form localized vacancies $\mathcal{V}_{\mathrm{O}}^{\text {loc }}$. (b) Structure of a split vacancy $\mathcal{V}_{\mathrm{O}}^{\text {split }}$ in the subsurface. (c) $\mathcal{V}_{\mathrm{O}}$ formation energies $E_{\mathrm{f}}^{\mathrm{O}}$ as a function of position with respect to the (001) surface. Values computed with different functionals are reported. The orange-shaded area highlights the energetic sink for $\mathcal{V}_{\mathrm{O}}^{\text {split }}$ in the subsurface (between positions 2 and 3 ). The bulk $E_{\mathrm{f}}^{\mathrm{O}}\left(\mathcal{V}_{\mathrm{O}}^{\text {loc }}\right.$ and $\left.\mathcal{V}_{\mathrm{O}}^{\text {split }}\right)$ is shown as dark shaded area on the right. Color code: $\mathrm{Bi}, \mathrm{V}$, and $\mathrm{O}$ are green, yellow, and red, respectively.

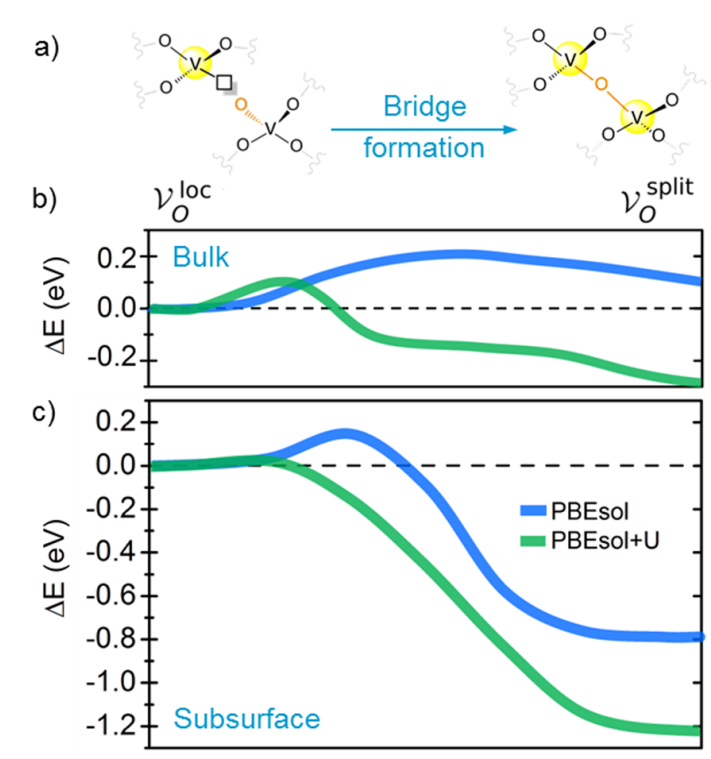

Figure 3. (a) Transition from localized $\left(\mathcal{V}_{\mathrm{O}}^{\text {loc }}\right)$ to split $\left(\mathcal{V}_{\mathrm{O}}^{\text {split }}\right)$ oxygen vacancy (b) in the bulk and (c) in the (001) subsurface of $\mathrm{BiVO}_{4}$. The energy profile connecting the two vacancy structures was obtained from NEB calculations with PBEsol and PBEsol+U functionals. $\Delta E$ on the ordinate represents the relative energy with respect to the localized vacancy. (typically greater than $\left.400{ }^{\circ} \mathrm{C}\right)^{2-4} \mathcal{V}_{\mathrm{O}}^{\text {loc }}$ and $\mathcal{V}_{\mathrm{O}}^{\text {split }}$ may interconvert. At higher $n$-type doping densities, the split vacancy $\mathcal{V}_{\mathrm{O}}^{\text {split }}$ may be favored, as its greater number of bonds allows for better stabilization of additional electrons. ${ }^{42}$

We now turn to the (001) surface, the thermodynamically most stable surface of $\mathrm{BiVO}_{4},{ }^{34,45}$ where $\mathcal{V}_{\mathrm{O}}$ 's are suggested to form quite easily. ${ }^{25}$ The (001) surface is cut parallel to the $\mathrm{Bi}-$ $\mathrm{V}$ layers such that the tetrahedral coordination of the $\mathrm{VO}_{4}$ tetrahedra is preserved and only 2 weaker $\mathrm{Bi}-\mathrm{O}$ bonds are broken, hence forming surface $\mathrm{BiO}_{6}{ }^{45}$ We have introduced oxygen vacancies in different surface and subsurface layers and calculated their formation energies $E_{\mathrm{f}}^{\mathrm{O}}$ as shown in Figure 2 and Table S4.

Upon relaxation the surface $\mathrm{Bi}-\mathrm{V}$ plane moves inward, decreasing the interplanar distance in the subsurface by $0.16 \AA$ without undergoing reconstruction. ${ }^{45,46}$ This compression leads to a higher density of $\mathrm{O}$ atoms in the subsurface, which in turn facilitates the removal of oxygen. This decreases the vacancy formation energy in the subsurface, i.e. in positions 2 and 3, as indicated in Figure 2. In contrast, the interlayer spacing in the subsubsurface increases, thus destabilizing oxygen vacancies at positions 4 and 5. A correlation of surface relaxation and oxygen vacancy formation energies is given in the Supporting Information (Table S5 and Figure S5). More importantly, the interplanar compression in the subsurface largely favors the formation a $\mathrm{V}-\mathrm{O}-\mathrm{V}$ bridge between surface and subsurface vanadiums upon $\mathrm{O}$ removal. The resulting $\mathcal{V}_{\mathrm{O}}^{\text {split }}$ configuration in the subsurface is stabilized by more than $1 \mathrm{eV}$, independent of the employed DFT functional (see orange-shaded area in Figure 2c), and most likely to be predominant. In other words, the (001) surface represents an energetic sink toward the formation of subsurface $\mathcal{V}_{\mathrm{O}}^{\text {split }}$. This finding is consistent with a recent report by Rossell et al. using local electron energy-loss spectroscopy, with which they observed reduced $\mathrm{V}$ sites on the $\mathrm{BiVO}_{4}$ surface. ${ }^{28}$ The accumulation of $\mathrm{O}$ vacancies close to the surface was also suggested by a recent photoemission study. ${ }^{47}$ The authors found that treating $\mathrm{BiVO}_{4}$ with oxygen plasma shifts the Fermi level downward and increases the workfunction, which could be due to reducing the number of $\mathcal{V}_{\mathrm{O}}$ 's near the surface. Our computed workfunctions show a similar behavior (Supporting Information, section S4.2.1). The presence of oxygen vacancies in the subsurface gives workfunctions of around 5.0-5.3 and $4.8-5.0 \mathrm{eV}$ for $\mathcal{V}_{\mathrm{O}}^{\text {loc }}$ and $\mathcal{V}_{\mathrm{O}}^{\text {split }}$, respectively, while the pristine, defect-free (001) surface possesses a workfunction of 6.2-6.4 eV (see Table S7).

When removing $\mathrm{O}$ in the subsurface and forming $\mathcal{V}_{\mathrm{O}}^{\text {split }}$, the surface $\mathrm{V}$ rotates, so that it builds a $\mathrm{V}-\mathrm{O}-\mathrm{V}$ bridge, by pointing one of its $\mathrm{V}-\mathrm{O}$ bonds up, perpendicular to the surface, as shown in Figure $2 \mathrm{~b}$. This further facilitates $\mathcal{V}_{\mathrm{O}}^{\text {split }}$ formation in contrast to the bulk where the $\mathrm{VO}_{4}$ unit needs to move to fill the empty coordination site at the adjacent $\mathrm{VO}_{3}$. Climbing image nudged elastic band (CI-NEB) calculations (Figure 3) show that in the bulk there is an energy barrier of $\sim 0.2 \mathrm{eV}(\sim 0.1 \mathrm{eV})$ for PBEsol (PBEsol+U) to be surmounted when going from $\mathcal{V}_{\mathrm{O}}^{\text {loc }}$ to $\mathcal{V}_{\mathrm{O}}^{\text {split }}$, whereas in the subsurface this transition is quasi-barrierless $(0.0 \mathrm{eV}$ for PBEsol, $0.1 \mathrm{eV}$ for PBEsol+U).

For the discussion of the electronic structure of the different types of vacancies we focus on hybrid functional results, which 

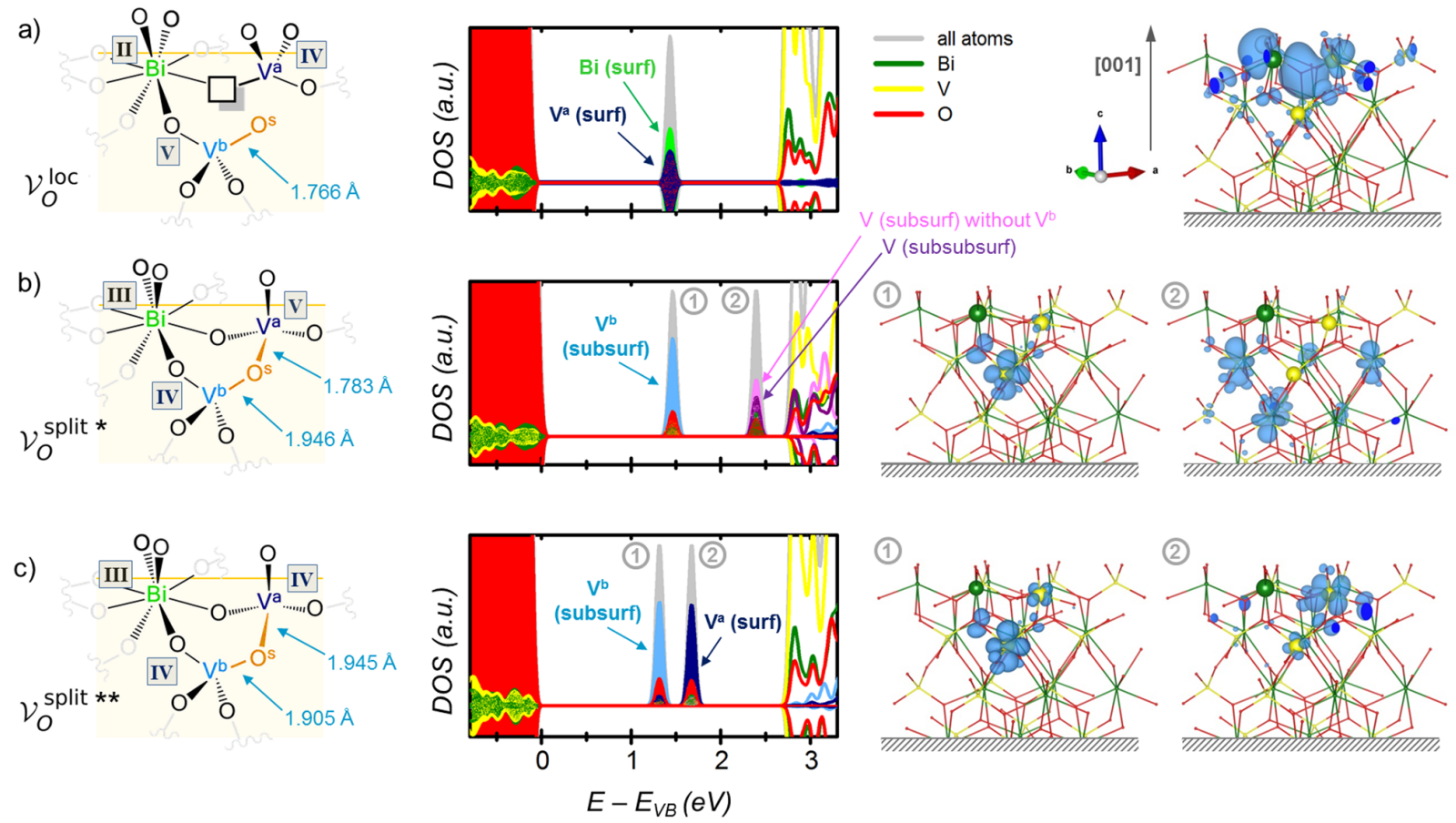

Figure 4. PBE0-10 results of chemical structures with relevant bond lengths and (formal) oxidation states, atom-projected density of states (aligned against the VBM; filled colors: occupied electronic states, empty colors: nonoccupied states; middle), and integrated local density of states (blue,

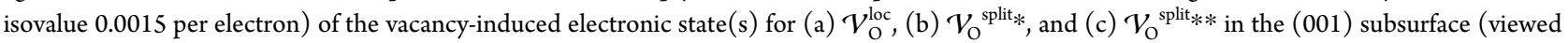
from the side).

typically provide a good description of the electronic properties of $\mathrm{BiVO}_{4}{ }^{12-14,35} \mathrm{~A}$ detailed discussion on the functional dependency is given in the Supporting Information (section 5). For the subsurface $\mathcal{V}_{\mathrm{O}}$ 's, we found three distinguishable configurations, while for the bulk even more electronic states with similar energies may be accessed. ${ }^{14}$ Those additional bulk states are further discussed in the Supporting Information, while herein we focus on the (001) subsurface. Figure 4 presents the geometric arrangements (including formal oxidation states) on the left, the projected densities of states (DOS) in the middle, and the spatial distributions of the vacancy-induced intragap state(s) on the right for the three configurations $\mathcal{V}_{\mathrm{O}}^{\text {loc }}$ (Figure 4a), $\mathcal{V}_{\mathrm{O}}^{\text {split } *}$

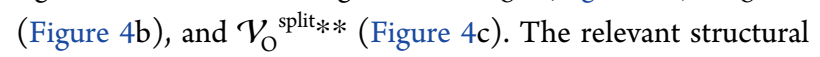
and electronic parameters for the subsurface are summarized in Table 1.

Localized $\mathcal{V}_{\mathrm{O}}^{\text {loc }} \mathrm{s}$ (Figure 4a) form vacancy-trapped electronic states: ${ }^{14}$ the excess electrons are trapped at the vacancy site, undercoordinated $\mathrm{VO}_{3}$ and $\mathrm{BiO}_{7}$ centers. Therefore, the adjacent $\mathrm{V}^{\mathrm{a}}$ gets reduced from $+\mathrm{V}$ to $+\mathrm{IV}$, and $\mathrm{Bi}$ from $+\mathrm{III}$ to +II, assuming classical oxidation states (Tables 1 and S8). The nonbonding electron pair induces a deep, doubly occupied singlet state $1.0-1.2 \mathrm{eV}$ below the CBM. This mixed electronic state is composed mainly of the $\mathrm{V}^{\mathrm{a}} \mathrm{t}_{2}$ set (with decreasing contributions: $\mathrm{d}_{y z}, \mathrm{~d}_{x y}, \mathrm{~d}_{x z}$ ) and Bi $\mathrm{p}_{x}$ and $\mathrm{p}_{z}$ orbitals, as shown in Figure $4 \mathrm{a}$ in the middle and on the right.

When forming the split vacancy $\mathcal{V}_{\mathrm{O}}^{\text {split }}$ in the subsurface (Figure $4 b, c)$, the two excess electrons are not trapped anymore as a bound pair but split onto different $\mathrm{V}$ centers, while $\mathrm{Bi}$ no longer bears additional electrons as its 6-fold surface coordination sphere is mostly re-established. We found
Table 1. Relevant Distances $d\left(\mathrm{~V}^{\mathrm{a}}-\mathrm{O}^{\mathrm{s}}\right)$ and $d\left(\mathrm{~V}^{\mathrm{b}}-\mathrm{O}^{\mathrm{s}}\right)$; Vacancy Formation Energies $E_{\mathrm{f}}^{\mathrm{O}}$; Position of Trap States with Respect to the Conduction Band Minimum (CBM); $E_{\text {CBM }}-E_{\text {trap }}$ (Singly or Doubly (d) Occupied); Bader Charges $(q) ;^{48}$ and Magnetizations $\mu$ on $\mathrm{V}^{\mathrm{a}}, \mathrm{V}^{\mathrm{b}}$, and $\mathrm{Bi}$ for the pristine (001) Surface and the Three $O$ Vacancy

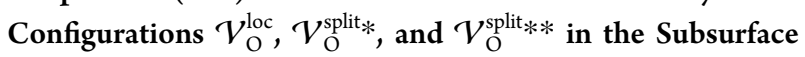
(at Position 2) Calculated with PBE0-10(*/**) $)^{a}$

\begin{tabular}{lllll} 
& $\begin{array}{c}\text { pristine } \\
\text { surface }\end{array}$ & \multicolumn{1}{c}{$\mathcal{V}_{\mathrm{O}}^{\text {loc }}$} & \multicolumn{1}{c}{$\mathcal{V}_{\mathrm{O}}^{\text {split* }}$} & $\mathcal{V}_{\mathrm{O}}^{\text {split** }}$ \\
& 2.894 & 2.224 & 1.783 & 1.945 \\
$d\left(\mathrm{~V}^{\mathrm{a}}-\mathrm{O}^{\mathrm{s}}\right)(\AA)$ & 1.762 & 1.766 & 1.946 & 1.905 \\
$d\left(\mathrm{~V}^{\mathrm{b}}-\mathrm{O}^{\mathrm{s}}\right)(\AA)$ & 4.348 & 3.767 & 2.295 & 2.259 \\
$d\left(\mathrm{Bi}^{\mathrm{a}}-\mathrm{O}^{\mathrm{s}}\right)(\AA)$ & & 3.98 & 3.08 & 3.04 \\
$E_{\mathrm{f}}^{\mathrm{O}}(\mathrm{eV})$ & & $1.22(\mathrm{~d})$ & $1.30,0.35$ & $1.36,1.00$ \\
$E_{\mathrm{CBM}}-E_{\text {trap }}(\mathrm{eV})$ & 2.20 & 1.89 & 2.18 & 2.09 \\
$q\left(\mathrm{~V}^{\mathrm{a}}\right) / \mathrm{lel}$ & 2.23 & 2.22 & 1.99 & 1.99 \\
$q\left(\mathrm{~V}^{\mathrm{b}}\right) / \mathrm{lel}$ & 2.08 & 1.56 & 2.00 & 2.01 \\
$q(\mathrm{Bi}) / \mathrm{lel}$ & 0.00 & 0.00 & 0.02 & 0.67 \\
$\mu\left(\mathrm{V}^{\mathrm{a}}\right) / \mu_{\mathrm{B}}$ & 0.00 & 0.00 & 0.64 & 0.66 \\
$\mu\left(\mathrm{V}^{\mathrm{b}}\right) / \mu_{\mathrm{B}}$ & 0.00 & 0.00 & 0.00 & 0.00 \\
$\mu(\mathrm{Bi}) / \mu_{\mathrm{B}}$ & & &
\end{tabular}

${ }^{a} \mathrm{O}^{\mathrm{s}}$ is the $\mathrm{O}$ atom coordinated to subsurface $\mathrm{V}^{\mathrm{b}}$ in pristine and $\mathcal{V}_{\mathrm{O}}^{\text {loc }}$ configurations.

two distinct $\mathcal{V}_{\mathrm{O}}^{\text {split }}$ configurations with equivalent energies, depending on whether the input geometry was taken from PBEsol (PBE0-10*) or PBEsol+U (PBE0-10**). In analogy to the calculation method, these are labeled $\mathcal{V}_{\mathrm{O}}^{\text {split* }}$ and $\mathcal{V}_{\mathrm{O}}^{\text {split***, }}$ respectively. We note here that the electronic configuration obtained with a semilocal GGA functional (PBEsol) is similar 
to $\mathcal{V}_{\mathrm{O}}^{\text {split* }}$ and the configuration calculated with $\mathrm{PBEsol}+\mathrm{U}$ is similar to $\mathcal{V}_{\mathrm{O}}^{\text {split**. Both }} \mathcal{V}_{\mathrm{O}}^{\text {split* }}$ and $\mathcal{V}_{\mathrm{O}}^{\text {split*** }}$ present a lower intragap state $1.30-1.35 \mathrm{eV}$ below the CBM mainly localized on the bridge forming subsurface $\mathrm{V}^{\mathrm{b}}$, which is therefore reduced from $+\mathrm{V}$ to $+\mathrm{IV}$. When forming the $\mathrm{V}^{\mathrm{a}}-\mathrm{O}^{\mathrm{s}}-\mathrm{V}^{\mathrm{b}}$ bridge, charge transfers from the surface $\mathrm{V}^{\mathrm{a}}$ to the subsurface $\mathrm{V}^{\mathrm{b}}$ where it becomes stabilized. This is not the case for the bulk, where $\mathrm{V}^{\mathrm{a}}$ and $\mathrm{V}^{\mathrm{b}}$ are equivalent (Supporting Information, section S4.1). The resulting electronic state is to the largest extent composed of the $\mathrm{V}^{\mathrm{b}} \mathrm{d}_{z}{ }^{2}$ orbital, which hybridizes with $\mathrm{d}_{x y}$ and $\mathrm{d}_{x^{2}-y^{2}}$ as the subsurface $\mathrm{V}$ loses its ideal tetrahedral symmetry, and has some contribution from adjacent oxygen atoms.

The difference of the two configurations $\left(\mathcal{V}_{\mathrm{O}}^{\mathrm{split}} * / * *\right)$ is given by the nature of the upper intragap state. For $\mathcal{V}_{\mathrm{O}}^{\text {split* }}$ this state is $0.3 \mathrm{eV}$ below the CBM and localized on different subsurface and subsubsurface $\mathrm{V}$ atoms, forming polaron-like states of $\mathrm{d}_{z^{2}}$ symmetry, similar to those observed in the bulk. ${ }^{14}$ For $\mathcal{V}_{\mathrm{O}}^{\text {split** }}$ the upper intragap state is much deeper inside the gap, $1.0 \mathrm{eV}$ below the CBM, and is localized on the bridgeforming, reduced surface $\mathrm{V}^{\mathrm{a} I \mathrm{~V}}$ with mixed d-orbital contributions. Isoenergetic singlet and triplet states were obtained for the split vacancy configurations. This suggests that the separated electrons interact so weakly that their spin-pairing energy is negligible.

The reason for the critical dependence of the electronic structure on the geometry may be explained by the variations in the relevant $\mathrm{V}-\mathrm{O}$ bond distances, which are given in Figure 4 and Table 1. Every time the excess electrons of the $\mathrm{O}$ vacancy are accepted by a $\mathrm{V}$ atom, they are placed in the $t_{2}$ (and e) orbital sets, which derive from tetrahedral splitting in the $\mathrm{VO}_{4}$ environment and which have a slightly antibonding character with respect to the $\mathrm{V}-\mathrm{O}$ bond. ${ }^{49}$ The shorter the V$\mathrm{O}$ distance, the larger the antibonding character, and therefore the higher the energy cost to populate the $3 \mathrm{~d}$ orbitals of the respective $\mathrm{V}$ atom. In the $\mathcal{V}_{\mathrm{O}}^{\text {split* }}$ geometry the $\mathrm{V}^{\mathrm{a}}-\mathrm{O}^{\mathrm{s}}$ bond length is only $1.783 \AA$ and $\mathrm{V}^{a}$ does not bear a significant amount of excess electron density, whereas the longer $\mathrm{V}^{\mathrm{b}}-\mathrm{O}^{\mathrm{s}}$ distance $\left(1.946 \AA\right.$ ) allows for electron localization at $\mathrm{V}^{\mathrm{b}}$. For

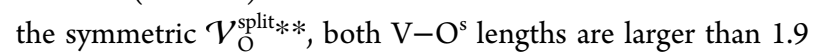
$\AA$ and the excess electron is accepted by both bridging $\mathrm{V}$ sites $\mathrm{V}^{\mathrm{a}}$ and $\mathrm{V}^{\mathrm{b}}$. It may also be argued vice versa, i.e. electron localization at $\mathrm{V}$ atoms leads to longer $\mathrm{V}-\mathrm{O}$ bond lengths because of populating antibonding states.

Because the $\mathcal{V}_{\mathrm{O}}^{\text {split* }}$ and $\mathcal{V}_{\mathrm{O}}^{\text {split** }}$ electronic states depend crucially on the bond distances, which constantly vary because of thermal motion at room temperature, it is reasonable to assume that both electronic configurations coexist in a thermal equilibrium under experimental conditions in the $\mathrm{BiVO}_{4}$ photoanode. $^{50}$ This means the second higher-energy electron is either localized on surface $\mathrm{V}^{\mathrm{a}}$ or delocalized in the (sub)subsurface. Similar to the latter $\mathcal{V}_{\mathrm{O}}^{\text {split* }}$, a two-dimensionally delocalized state has recently been reported for the $\mathrm{WO}_{3}$ surface and is expected to be favorable for water oxidation catalysis. ${ }^{50}$ It may lead to enhanced electronic conduction parallel to the (001) surface and therefore contribute favorably to $n$-type conduction. Anisotropic conduction within the $\mathrm{Bi}-\mathrm{V}$ planes was indeed experimentally measured. ${ }^{9}$ The favored stabilization of $\mathcal{V}_{\mathrm{O}}$ in the subsurface and the two-dimensionally extended charge delocalization could also explain experimentally observed preferential charge separation along the [001] crystal direction and accumulation of charges at the (001) surface. $^{51,52}$

To further understand the implications of the different subsurface $\mathcal{V}_{\mathrm{O}}$ states, we also analyzed the layer-resolved DOS (Figures S7 and S8). ${ }^{53} \mathrm{We}$ see that the contribution of the surface layer to the valence band maximum is slightly reduced when creating $\mathcal{V}_{\mathrm{O}}^{\text {split }}$, as compared to the pristine surface and $\mathcal{V}_{\mathrm{O}}^{\text {loc }}$. The surface layer does not significantly contribute to the CBM, which aids photooxidation because photoexcited electrons tend to go to the bulk and not to the surface. A more detailed discussion is given in the Supporting Information (section S4.2.2).

Taken together, we suggest that oxygen vacancies in $\mathrm{BiVO}_{4}$ do increase conductivity. In the bulk, $\mathrm{O}$ removal allows for a reversible formation of $\mathrm{V}-\mathrm{O}-\mathrm{V}$ bridges, which increases both electronic and ionic conduction: Partial electron delocalization in $\mathcal{V}_{\mathrm{O}}^{\text {split }}$ leads to polaronic ${ }^{14,15}$ or delocalized charged states at the bottom of the conduction band (Figure S6), which promote electron transport. Further, the moderately low transition barriers for consecutive forming $(\lesssim 0.2 \mathrm{eV})$ and breaking $(\lesssim 0.4 \mathrm{eV})$ of $\mathrm{V}_{2} \mathrm{O}_{7}$ from neighboring $\mathrm{VO}_{3}$ and $\mathrm{VO}_{4}$ (Figure $3 \mathrm{~b}$ ) units may enable ionic conduction through oxygen migration (Supporting Information, section S5.5) ${ }^{40}$ Considering the (001) surface, $\mathcal{V}_{\mathrm{O}}^{\text {split, }}$, accumulate in the subsurface where they are highly stabilized and formed without barrier. The large energy barrier $(\sim 1 \mathrm{eV}$; Figure $3 \mathrm{c})$ to the breaking of $\mathrm{V}_{2} \mathrm{O}_{7}$ in the subsurface acts as a trap for oxygen vacancies. As such, electrons transfer from the surface plane $\left(\mathrm{V}^{\mathrm{a}}\right)$ to the subsurface and sub-subsurface where they partially delocalize, leading to enhanced electronic conduction parallel to the (001) facet. $^{51}$

In conclusion, two different types of oxygen vacancies were found in $\mathrm{BiVO}_{4}$ : one forming a vacancy-trapped charge state with the two surplus electrons paired and localized at the vacancy site, the other quenching the vacancy by bridging V$\mathrm{O}-\mathrm{V}$ and concomitantly delocalizing one electron. While in the bulk both structures are almost isoenergetic and may coexist at thermal equilibrium, the (001) subsurface presents a large energetic sink for the creation of $\mathrm{V}-\mathrm{O}-\mathrm{V}$ units. This may enhance electronic conduction parallel to the (001) surface. We investigated the impact of vacancies on the electronic properties of $\mathrm{BiVO}_{4}$ for the first time, not only in bulk but also on the most relevant surface, and show that the defective surface is a very promising candidate to explain the photochemical properties of $\mathrm{BiVO}_{4}$.

\section{ASSOCIATED CONTENT}

S Supporting Information

Computational methods, geometrical and electronic structures, vacancy formation energies, relevant energies (such as band gaps, trap state energies, and work functions), and a detailed functional comparison (PDF)

\section{AUTHOR INFORMATION}

\section{Corresponding Authors}

*E-mail: nlopez@iciq.es.

*E-mail: aselloni@princeton.edu. 


\section{ORCID $\odot$}

Franziska Simone Hegner: 0000-0002-7645-4418

Daniel Forrer: 0000-0002-1969-3842

José Ramón Galán-Mascarós: 0000-0001-7983-9762

Núria López: 0000-0001-9150-5941

Annabella Selloni: 0000-0001-5896-3158

Notes

The authors declare no competing financial interest.

All structures can be found at the ioChemBD database under the DOI $10.19061 /$ iochem-bd-1-133. ${ }^{37}$

\section{ACKNOWLEDGMENTS}

The authors thank the groups of Prof. S. Gimenez and Prof. J. Durrant, in particular S. Selim, for fruitful discussions about oxygen vacancy defects. This work was funded by the European Union's Horizon 2020 project A-LEAF (Grant Agreement No. 732840); the Spanish Ministerio de Ciencia, Innovación y Universidades (RTI2018-101394-BI00); and the "LaCaixa" -Severo Ochoa International Programme of Ph.D. Scholarships for F.S.H.'s predoctoral grant. The authors further thank the Barcelona Supercomputaing Centre (BSC-RES) for generous computational resources. A. S. acknowledges the support of DoE-BES Division of Chemical Sciences, Geosciences and Biosciences under Award DE-SC0007347.

\section{REFERENCES}

(1) Kudo, A.; Ueda, K.; Kato, H.; Mikami, I. Photocatalytic $\mathrm{O}_{2}$ Evolution under Visible Light Irradiation on $\mathrm{BiVO}_{4}$ in Aqueous $\mathrm{AgNO}_{3}$ Solution. Catal. Lett. 1998, 53, 229-230.

(2) Sharp, I. D.; Cooper, J. K.; Toma, F. M.; Buonsanti, R. Bismuth Vanadate as a Platform for Accelerating Discovery and Development of Complex Transition-Metal Oxide Photoanodes. ACS Energy Lett. 2017, 2, 139-150.

(3) Tan, H. L.; Amal, R.; Ng, Y. H. Alternative Strategies in Improving the Photocatalytic and Photoelectrochemical Activities of Visible Light-Driven $\mathrm{BiVO}_{4}$ : A Review. J. Mater. Chem. A 2017, 5, 16498-16521.

(4) Tolod, K.; Hernández, S.; Russo, N. Recent Advances in the $\mathrm{BiVO}_{4}$ Photocatalyst for Sun-Driven Water Oxidation: Top-Performing Photoanodes and Scale-Up Challenges. Catalysts 2017, 7, 13.

(5) Pihosh, Y.; Turkevych, I.; Mawatari, K.; Uemura, J.; Kazoe, Y.; Kosar, S.; Makita, K.; Sugaya, T.; Matsui, T.; Fujita, D.; et al. Photocatalytic Generation of Hydrogen by Core-Shell $\mathrm{WO}_{3} / \mathrm{BiVO}_{4}$ Nanorods with Ultimate Water Splitting Efficiency. Sci. Rep. 2015, 5, 11141.

(6) Ma, Y.; Pendlebury, S. R.; Reynal, A.; Le Formal, F.; Durrant, J. R. Dynamics of Photogenerated Holes in Undoped $\mathrm{BiVO}_{4}$ Photoanodes for Solar Water Oxidation. Chem. Sci. 2014, 5, 2964-2973.

(7) Zachäus, C.; Abdi, F. F.; Peter, L. M.; van de Krol, R. Photocurrent of $\mathrm{BiVO}_{4}$ Is Limited by Surface Recombination, Not Surface Catalysis. Chem. Sci. 2017, 8, 3712-3719.

(8) Abdi, F. F.; Savenije, T. J.; May, M. M.; Dam, B.; van de Krol, R The Origin of Slow Carrier Transport in $\mathrm{BiVO}_{4}$ Thin Film Photoanodes: A Time-Resolved Microwave Conductivity Study. J. Phys. Chem. Lett. 2013, 4, 2752-2757.

(9) Rettie, A. J. E.; Lee, H. C.; Marshall, L. G.; Lin, J.-F.; Capan, C.; Lindemuth, J.; Mccloy, J. S.; Zhou, J.; Bard, A. J.; Mullins, C. B. Combined Charge Carrier Transport and Photoelectrochemical Characterization of $\mathrm{BiVO}_{4}$ Single Crystals: Intrinsic Behavior of a Complex Metal Oxide. J. Am. Chem. Soc. 2013, 135, 11389-11396.

(10) Rettie, A. J.; Chemelewski, W. D.; Emin, D.; Mullins, C. B. Unravelling Small-Polaron Transport in Metal Oxide Photoelectrodes. J. Phys. Chem. Lett. 2016, 7, 471-479.

(11) Ziwritsch, M.; Muller, S.; Hempel, H.; Unold, T.; Abdi, F. F.; van de Krol, R.; Friedrich, D.; Eichberger, R. Direct Time-Resolved
Observation of Carrier Trapping and Polaron Conductivity in $\mathrm{BiVO}_{4}$. ACS Energy Lett. 2016, 1, 888-894.

(12) Kweon, K. E.; Hwang, G. S.; Kim, J.; Kim, S.; Kim, S. Electron Small Polarons and Their Transport in Bismuth Vanadate: A First Principles Study. Phys. Chem. Chem. Phys. 2015, 17, 256-260.

(13) Wiktor, J.; Ambrosio, F.; Pasquarello, A. Role of Polarons in Water Splitting: The Case of $\mathrm{BiVO}_{4}$. ACS Energy Lett. 2018, 3, 16931697.

(14) Seo, H.; Ping, Y.; Galli, G. Role of Point Defects in Enhancing the Conductivity of $\mathrm{BiVO}_{4}$. Chem. Mater. 2018, 30, 7793-7802.

(15) Wu, F.; Ping, Y. Combining Landau-Zener Theory and Kinetic Monte Carlo Sampling for Small Polaron Mobility of Doped $\mathrm{BiVO}_{4}$ from First-Principles. J. Mater. Chem. A 2018, 6, 20025-20036.

(16) Liang, Y.; Tsubota, T.; Mooij, L. P.; van de Krol, R. Highly Improved Quantum Efficiencies for Thin Film $\mathrm{BiVO}_{4}$ Photoanodes. J. Phys. Chem. C 2011, 115, 17594-17598.

(17) Li, D.; Wang, W.; Jiang, D.; Zheng, Y.; Li, X. Surfactant-Free Hydrothermal Fabrication of Monoclinic $\mathrm{BiVO}_{4}$ Photocatalyst with Oxygen Vacancies by Copper Doping. RSC Adv. 2015, 5, 1437414381.

(18) Wang, S.; Chen, P.; Bai, Y.; Yun, J.-H.; Liu, G.; Wang, L. New $\mathrm{BiVO}_{4}$ Dual Photoanodes with Enriched Oxygen Vacancies for Efficient Solar-Driven Water Splitting. Adv. Mater. 2018, 30, 1800486.

(19) Vinke, I. C.; Diepgrond, J.; Boukamp, B. A.; De Vries, K. J.; Burggraaf, A. J. Bulk and Electrochemical Properties of $\mathrm{BiVO}_{4}$. Solid State Ionics 1992, 57, 83-89.

(20) Wang, G.; Ling, Y.; Li, Y. Oxygen-Deficient Metal Oxide Nanostructures for Photoelectrochemical Water Oxidation and Other Applications. Nanoscale 2012, 4, 6682-6691.

(21) Yin, W. J.; Wei, S. H.; Al-Jassim, M. M.; Turner, J.; Yan, Y. Doping Properties of Monoclinic $\mathrm{BiVO}_{4}$ Studied by First-Principles Density-Functional Theory. Phys. Rev. B: Condens. Matter Mater. Phys. 2011, 83, 155102.

(22) Yuan, Y.; Huang, Y.; Ma, F.; Zhang, Z.; Wei, X. Effects of Oxygen Vacancy on the Mechanical, Electronic and Optical Properties of Monoclinic $\mathrm{BiVO}_{4}$. J. Mater. Sci. 2017, 52, 8546-8555.

(23) Freysoldt, C.; Grabowski, B.; Hickel, T.; Neugebauer, J.; Kresse, G.; Janotti, A.; Van de Walle, C. G. First-Principles Calculations for Point Defects in Solids. Rev. Mod. Phys. 2014, 86, 253

(24) Hu, J.; Zhao, X.; Chen, W.; Su, H.; Chen, Z. Theoretical Insight into the Mechanism of Photoelectrochemical Oxygen Evolution Reaction on $\mathrm{BiVO}_{4}$ Anode with Oxygen Vacancy. J. Phys. Chem. C 2017, 121, 18702-18709.

(25) Zhao, X.; Hu, J.; Yao, X.; Chen, S.; Chen, Z. Clarifying the Roles of Oxygen Vacancy in W-doped $\mathrm{BiVO}_{4}$ for Solar Water Splitting. ACS Appl. Energy Mater. 2018, 1, 3410-3419.

(26) Kho, Y. K.; Teoh, W. Y.; Iwase, A.; Mädler, L.; Kudo, A.; Amal, R. Flame Preparation of Visible-Light-Responsive $\mathrm{BiVO}_{4}$ Oxygen Evolution Photocatalysts with Subsequent Activation Via Aqueous Route. ACS Appl. Mater. Interfaces 2011, 3, 1997-2004.

(27) Cooper, J. K.; Scott, S. B.; Ling, Y.; Yang, J.; Hao, S.; Li, Y.; Toma, F. M.; Stutzmann, M.; Lakshmi, K. V.; Sharp, I. D. Role of Hydrogen in Defining the $n$-Type Character of $\mathrm{BiVO}_{4}$ Photoanodes. Chem. Mater. 2016, 28, 5761-5771.

(28) Rossell, M. D.; Agrawal, P.; Borgschulte, A.; Hebert, C.; Passerone, D.; Erni, R. Direct Evidence of Surface Reduction in Monoclinic $\mathrm{BiVO}_{4}$. Chem. Mater. 2015, 27, 3593-3600.

(29) Wang, G.; Ling, Y.; Lu, X.; Qian, F.; Tong, Y.; Zhang, J. Z.; Lordi, V.; Rocha Leao, C.; Li, Y. Computational and Photoelectrochemical Study of Hydrogenated Bismuth Vanadate. J. Phys. Chem. C 2013, 117, 10957-10964.

(30) Kim, T. W.; Ping, Y.; Galli, G. A.; Choi, K.-S. Simultaneous Enhancements in Photon Absorption and Charge Transport of Bismuth Vanadate Photoanodes for Solar Water Splitting. Nat. Commun. 2015, 6, 9769.

(31) Ullah, H.; Tahir, A. A.; Mallick, T. K. Structural and Electronic Properties of Oxygen Defective and Se-doped $p$-Type $\mathrm{BiVO}_{4}(001)$ 
Thin Film for the Applications of Photocatalysis. Appl. Catal., B 2018, 224, 895-903.

(32) Giannozzi, P.; Baroni, S.; Bonini, N.; Calandra, M.; Car, R.; Cavazzoni, C.; Ceresoli, D.; Chiarotti, G. L.; Cococcioni, M.; Dabo, I.; et al. QUANTUM ESPRESSO: A Modular and Open-source Software Project for Quantum Simulations of Materials. J. Phys.: Condens. Matter 2009, 21, 395502.

(33) Giannozzi, P.; Andreussi, O.; Brumme, T.; Bunau, O.; Nardelli, M. B.; Calandra, M.; Car, R.; Cavazzoni, C.; Ceresoli, D.; Cococcioni, M.; et al. Advanced Capabilities for Materials Modelling with QUANTUM ESPRESSO. J. Phys.: Condens. Matter 2017, 29, 465901. (34) Hu, J.; Chen, W.; Zhao, X.; Su, H.; Chen, Z. Anisotropic Electronic Characteristics, Adsorption, and Stability of Low-Index $\mathrm{BiVO}_{4}$ Surfaces for Photoelectrochemical Applications. ACS Appl. Mater. Interfaces 2018, 10, 5475-5484.

(35) Hegner, F. S.; Herraiz-Cardona, I.; Cardenas-Morcoso, D.; López, N.; Galán-Mascarós, J.-R.; Gimenez, S. Cobalt Hexacyanoferrate on $\mathrm{BiVO}_{4}$ Photoanodes for Robust Water Splitting. ACS Appl. Mater. Interfaces 2017, 9, 37671-37681.

(36) Álvarez-Moreno, M.; de Graaf, C.; Lopez, N.; Maseras, F.; Poblet, J. M.; Bo, C. Managing the Computational Chemistry Big Data Problem: The ioChem-BD Platform. J. Chem. Inf. Model. 2015, $55,95-103$

(37) Hegner, F. S. Link to ioChem-BD: Oxygen-vacancies $\mathrm{BiVO}_{4}$. 2016; DOI: 10.19061/iochem-bd-1-133.

(38) The notation of crystal facets/directions depends on the definition of the crystal axes. We define here the $c$-axis as the principal axis. In several other studies, the $b$-axis is defined as the principal axis, and in those studies (010) is the most stable crystal facet.

(39) Momma, K.; Izumi, F. VESTA: A Three-dimensional Visualization System for Electronic and Structural Analysis. J. Appl. Crystallogr. 2008, 41, 653-658.

(40) Yang, X.; Fernández-Carrión, A. J.; Wang, J.; Porcher, F.; Fayon, F.; Allix, M.; Kuang, X. Cooperative Mechanisms of Oxygen Vacancy Stabilization and Migration in the Isolated Tetrahedral Anion Scheelite Structure. Nat. Commun. 2018, 9, 4484.

(41) Greenwood, N. N.; Earnshaw, A. Chemistry of the Elements; Elsevier Science: Amsterdam, The Netherlands, 2012.

(42) Centoni, S. A.; Sadigh, B.; Gilmer, G. H.; Lenosky, T. J.; de la Rubia, T. D.; Musgrave, C. B. First-Principles Calculation of Intrinsic Defect Formation Volumes in Silicon. Phys. Rev. B: Condens. Matter Mater. Phys. 2005, 72, 195206.

(43) Seebauer, E. G.; Kratzer, M. C. Charged Point Defects in Semiconductors. Mater. Sci. Eng., R 2006, 55, 57-149.

(44) Ganduglia-Pirovano, M. V.; Sauer, J. Stability of Reduced $\mathrm{V}_{2} \mathrm{O}_{5}$ (001) Surfaces. Phys. Rev. B: Condens. Matter Mater. Phys. 2004, 70, No. 045422.

(45) Zhao, Z.; Li, Z.; Zou, Z. Structure and Energetics of Low-index Stoichiometric Monoclinic Clinobisvanite $\mathrm{BiVO}_{4}$ Surfaces. RSC Adv. 2011, 1, 874.

(46) Favaro, M.; Uecker, R.; Nappini, S.; Pis, I.; Magnano, E.; Bluhm, H.; van de Krol, R.; Starr, D. E. Chemical, Structural, and Electronic Characterization of the (010) Surface of Single Crystalline Bismuth Vanadate. J. Phys. Chem. C 2019, 123, 8347-8359.

(47) Hermans, Y.; Murcia-López, S.; Klein, A.; Van de Krol, R.; Andreu, T.; Morante, J. R.; Toupance, T.; Jaegermann, W. Analysis of the Interfacial Characteristics of $\mathrm{BiVO}_{4} /$ Metal Oxide Heterostructures and Its Implication on Their Junction Properties. Phys. Chem. Chem. Phys. 2019, 21, 5086-5096.

(48) Henkelman, G.; Arnaldsson, A.; Jónsson, H. A Fast and Robust Algorithm for Bader Decomposition of Charge Density. Comput. Mater. Sci. 2006, 36, 354-360.

(49) Dolgos, M. R.; Paraskos, A. M.; Stoltzfus, M. W.; Yarnell, S. C.; Woodward, P. M. The Electronic Structures of Vanadate Salts: Cation Substitution as a Tool for Band Gap Manipulation. J. Solid State Chem. 2009, 182, 1964-1971.

(50) Gerosa, M.; Gygi, F.; Govoni, M.; Galli, G. The Role of Defects and Excess Surface Charges at Finite Temperature for Optimizing Oxide Photoabsorbers. Nat. Mater. 2018, 17, 1122-1127.
(51) Li, R.; Zhang, F.; Wang, D.; Yang, J.; Li, M.; Zhu, J.; Zhou, X.; Han, H.; Li, C. Spatial Separation of Photogenerated Electrons and Holes Among $\{010\}$ and $\{110\}$ Crystal Facets of $\mathrm{BiVO}_{4}$. Nat. Commun. 2013, 4, 1432

(52) Han, H. S.; Shin, S.; Kim, D. H.; Park, I. J.; Kim, J. S.; Huang, P.-S.; Lee, J.-K.; Cho, I. S.; Zheng, X. Boosting the Solar Water Oxidation Performance of a $\mathrm{BiVO}_{4}$ Photoanode by Crystallographic Orientation Control. Energy Environ. Sci. 2018, 11, 1299-1306.

(53) Selcuk, S.; Selloni, A. Facet-dependent Trapping and Dynamics of Excess Electrons at Anatase $\mathrm{TiO}_{2}$ Surfaces and Aqueous Interfaces. Nat. Mater. 2016, 15, 1107. 